\title{
P-1176- Examination Of Oral Glucose Tolerance Test Results Adults In Eastern Anatolia, Turkey
}

\author{
Doğan Nasır Binici', Özge Timur¹, Aykut Turhan¹, Pınar Tosun Taşar², Mehmet Emin Budak \\ ${ }^{1}$ Region Training and Research Hospital, Department of Internal Medicine, Erzurum, TURKEY \\ ${ }^{2}$ Region Training and Research Hospital, Department of Geriatrics, Erzurum, TURKEY
}

\section{INTRODUCTION:}

Diabetes is a growing concern health problem for the whole world. Diabetes may be diagnosed on fasting plasma glucose (FPG) or 2-h plasma glucose (2-h PG) value after a $75 \mathrm{~g}$ oral glucose test (OGTT) or A1C criteria.

2-h-PG is an appropriate diagnostic testing.Impaired fasting glucose (IFG) is defined as FPG levels between $100-126 \mathrm{mg} / \mathrm{dl}$ and impaired glucose rolerance (IGT) as 2-h PG after $75 \mathrm{~g}$ OGTT levels between 140-199mg/dl. 2-h PG after $75 \mathrm{~g}$ OGTT levels $\leq 200 \mathrm{mg} / \mathrm{dl}$ indicates the diagnosis of diabetes. IFG+IGT is defined as FPG levels between 100-126mg/dl and 2-h PG after $75 \mathrm{~g}$ OGTT levels between 140-199mg/dl.

OGTT results of 790 adults were examined in this study.

\section{METHODS:}

In this study, we examined the OGTT results of 790 individuals attending to our outpatient clinic. Oral glucose tolerance test was applied to patients with a fasting blood glucose of $100-126 \mathrm{mg} /$ $\mathrm{dl}$, obese, unexplained retinopathy, neuropathy and coronary disease or a history of gestational diabetes.

\section{RESULTS:}

The average age of $256(32,4 \%)$ male $534(67,6 \%)$ female patients is $46.92 \pm 14,68.164(20,7 \%)$ patients were defined as IFG (111 female, 53 male), 119 (15\%) IGT (96 female, 33 male), $161(20,4 \%)$ IFG+IGT ( 108 female, 53 male). 228 (28,9\%) were diagnosed as diabetes (158 female, 70 male). 312 (39,5\%) patients had normal OGTT testing (228 female, 90 male).

\begin{tabular}{|l|c|c|}
\hline & Female & Male \\
\hline IFG & 111 & 53 \\
\hline IGT & 96 & 33 \\
\hline IFG+IGT & 108 & 53 \\
\hline Diabetes & 158 & 70 \\
\hline Normal & 228 & 90 \\
\hline
\end{tabular}

\section{CONCLUSION:}

The prevalence of diabetes in our country is over $10 \%$ in individuals over the age of 40 . For this reason, diabetes screening is recommended for all individuals over 40 years of age regardless of their weight. All asymptomatic individuals who are overweight or obese and have risk factors should be investigated for diabetes. IFG and IGT are accepted as prediabetes. Both are risk factors for diabetes and cardiovascular disease. It has been shown that $5-10 \%$ of the prediabetic patients go to the diabetes stage per year. Approximately $70 \%$ of prediabetic patients who are followed for life time are diabetic in the later stages of their life. Combined IFG + IGT refers to further impairment of glucose metabolism. As a result of our study, both diabetic and prediabetic conditions were determined and these patients were informed and followed up.

Therefore, although OGTT is more difficult and cost-effective, is very important to diagnose and prevent health problems that may occur in the future.

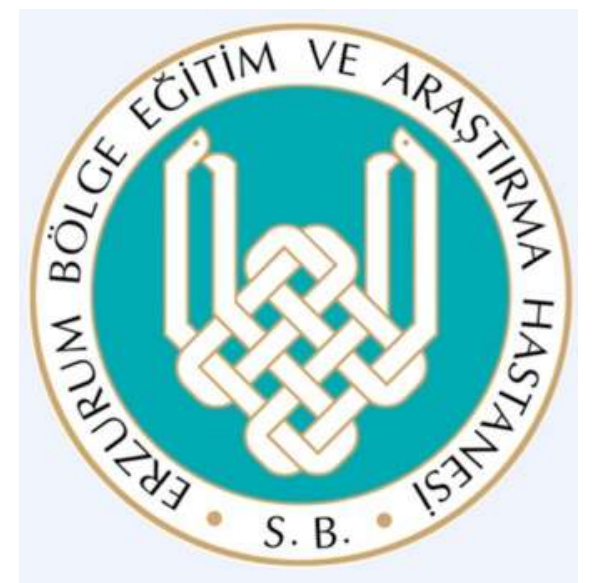

